\title{
Improved Approach to Robust Control for Type-2 T-S Fuzzy Systems
}

\author{
Bum Yong Park ${ }^{1}{ }^{1}$ and JaeWook Shin $\mathbb{D D}^{2}$ \\ ${ }^{1}$ Division of Electrical Engineering, Kumoh National Institute of Technology, Gumi-si, Gyeongsangbuk-do, Republic of Korea \\ ${ }^{2}$ Department of Medical and Mechatronics Engineering, Soonchunhyang University, Asan, Chungnam, Republic of Korea \\ Correspondence should be addressed to Bum Yong Park; bumyong.park@kumoh.ac.kr
}

Received 24 November 2017; Accepted 26 February 2018; Published 29 March 2018

Academic Editor: Haipeng Peng

Copyright ( 2018 Bum Yong Park and JaeWook Shin. This is an open access article distributed under the Creative Commons Attribution License, which permits unrestricted use, distribution, and reproduction in any medium, provided the original work is properly cited.

\begin{abstract}
This paper is concerned with the robust stability conditions to stabilize the type 2 Takagi-Sugeno (T-S) fuzzy systems. The conditions effectively handle parameter uncertainties using lower and upper membership functions. To improve the solvability of the stability conditions, we establish a multigain controller with comprehensive information of the lower and upper membership grades. In addition, a well-organized relaxation technique is proposed to fully exploit relationship among fuzzy weighting functions and their lower and upper membership grades, which enlarges a set of feasible solutions. Therefore, we derive a less conservative stabilization condition in terms of linear matrix inequalities (LMIs) than those in the literature. Two simulation examples illustrate the effectiveness and robustness of the derived stabilization conditions.
\end{abstract}

\section{Introduction}

Over the past few decades, the type 1 Takagi-Sugeno (T-S) fuzzy model has attracted much attention because it can systematically represent nonlinear systems via an interpolation method that smoothly connects some local linear systems based on fuzzy weighting functions [1-3]. The main advantage of type 1 T-S fuzzy systems is that they allow us to apply the well-established linear system theory for the analysis and synthesis of nonlinear systems. For this reason, the type $1 \mathrm{~T}-\mathrm{S}$ fuzzy model has been a popular choice not only in consumer products but also in industrial processes, such as power converters [4], motors [5], and solar power generator systems [6].

For the stability analysis and synthesis of type 1 fuzzy control systems, Lyapunov stability theory is widely used [7-10]. Fundamental stability conditions in terms of linear matrix inequalities (LMIs) are derived from Lyapunov stability condition. The conditions guarantee the stability of the fuzzy control systems if there exists a solution to a set of LMIs. Many researchers introduced the stability conditions and relaxed stability conditions using parallel distributed compensation (PDC) concept [8]. Using the information of type 1 fuzzy membership functions, the stability conditions can be further relaxed [11-14].

Although the type 1 fuzzy control system can effectively handle the nonlinear systems, it cannot guarantee the stability of the nonlinear systems with parameter uncertainties. Recently, type 2 fuzzy systems have attracted a lot of research attention [15] because they are better at handling uncertainties than the conventional type 1 fuzzy systems [16, 17]. Hence, for the stability analysis and controller synthesis of nonlinear systems with parameter uncertainties, it is essential to use type 2 fuzzy systems. Several researchers have researched such type 2 fuzzy systems [18-20]. However, all the aforementioned papers have seldom studied stability analysis and controller synthesis for type 2 T-S fuzzy systems. This motivates the study of the stability analysis and controller synthesis of type 2 T-S fuzzy systems.

Recently, some researchers have studied stability analysis and controller synthesis for type 2 T-S fuzzy systems [21-25]. In [21], an interval type 2 T-S fuzzy controller was proposed using a common controller gain that collectively depends on the sum of lower upper membership grades. In [22], the controller design for the interval type 2 T-S fuzzy system 
was introduced using a membership function different from a membership function of the system.

In the above studies based on the type $2 \mathrm{~T}$-S fuzzy systems, the stability conditions for the design of the type 2 fuzzy controller have some tuning parameters, which can result in increasing the implementation effort. It motivates the study of the controller synthesis for type 2 T-S fuzzy system. This paper studies the robust stability conditions to stabilize type 2 T-S fuzzy systems. The conditions effectively handle parameter uncertainties using lower and upper membership functions. To improve the solvability of the stability conditions, we establish a multigain controller with comprehensive information of the lower and upper membership grades. In addition, we propose a well-organized relaxation technique that fully exploits relationship among fuzzy weighting functions and their lower and upper membership grades, which enlarges a set of feasible solutions. Therefore, we derive a less conservative stabilization condition in terms of LMIs than those in the literature. The proposed condition has a simple structure without tuning parameters. Finally, two simulation examples are given to illustrate the effectiveness and robustness of the derived stabilization condition.

Notation. The notations $X \geq Y$ and $X>Y$ mean that $X-Y$ is positive semidefinite and positive definite, respectively. In symmetric block matrices, $(*)$ is used as an ellipsis for terms that are induced by symmetry. Furthermore, $\operatorname{Sym}(X)=X+$ $X^{T}$ stands for any matrix $X$.

\section{System Description and Preliminaries}

Let us consider the following type 2 T-S fuzzy model [21] that represents a continuous-time nonlinear system: for $i \in \mathscr{R}=$ $\{1,2, \ldots, p\}$

Plant Rule $i$ : IF $f_{1}(x(t))$ is $\widetilde{\mathscr{F}}_{i 1}$ and $\ldots f_{s}(x(t))$ is $\widetilde{\mathscr{F}}_{i s}$,

THEN $\dot{x}(t)=A_{i} x(t)+B_{i} u(t)$,

where $x(t) \in \mathscr{R}^{n}, u(t) \in \mathscr{R}^{m}$ denote the state and control input, respectively; $\widetilde{\mathscr{F}}_{i j}$ denotes a type 2 fuzzy set of rules $i$ corresponding to the function $f_{j}(x(t))$; and $p$ denotes the number of IF-THEN rules. The firing interval of the $i$ th rule is as follows:

$$
\begin{aligned}
& \widetilde{\theta}_{i}(x(t))=\left[\underline{\mu}_{\widetilde{F}_{i 1}}\left(f_{1}(x(t))\right) \times \cdots\right. \\
& \quad \times \underline{\mu}_{\widetilde{F}_{i s}}\left(f_{s}(x(t))\right), \bar{\mu}_{\widetilde{F}_{i 1}}\left(f_{1}(x(t))\right) \times \cdots \\
& \left.\quad \times \bar{\mu}_{\widetilde{F}_{i s}}\left(f_{s}(x(t))\right)\right] \equiv\left[\theta_{i}^{L}(x(t)), \theta_{i}^{U}(x(t))\right],
\end{aligned}
$$

where $\underline{\mu}_{\widetilde{F}_{i j}}\left(f_{j}(x(t))\right) \in[0,1]$ and $\bar{\mu}_{\widetilde{F}_{i j}}\left(f_{j}(x(t))\right) \in[0,1]$ denote the lower and upper grades of membership associated with the lower and upper membership functions, respectively. Here, $\underline{\underline{F}}_{\widetilde{F}_{i j}}\left(f_{j}(x(t))\right) \leq \bar{\mu}_{\widetilde{F}_{i j}}\left(f_{j}(x(t))\right)$, which leads to
$\theta_{i}^{L}(x(t)) \leq \theta_{i}^{U}(x(t))$ for all $i$. The overall type 2 T-S fuzzy system is inferred as follows:

$$
\dot{x}(t)=\sum_{i=1}^{p} \theta_{i}(x(t))\left(A_{i} x(t)+B_{i} u(t)\right)
$$

where $\theta_{i}(x(t))=\eta(x(t)) \theta_{i}^{L}(x(t))+(1-\eta(x(t))) \theta_{i}^{U}(x(t))$ denotes a fuzzy weighting function in which $\eta(x(t)) \in[0,1]$ is a nonlinear function and not necessary to be considered in this paper. Now, consider a multigain controller that is individually dependent on the lower and upper membership grades such as

$$
u(t)=\sum_{j=1}^{p}\left(\underline{\theta}_{j}(x(t)) \underline{K}_{j}+\bar{\theta}_{j}(x(t)) \bar{K}_{j}\right) x(t),
$$

where

$$
\begin{aligned}
& \underline{\theta}_{j}(x(t)) \triangleq \frac{\theta_{j}^{L}(x(t))}{\sum_{l=1}^{p}\left(\theta_{l}^{L}(x(t))+\theta_{l}^{U}(x(t))\right)}, \\
& \bar{\theta}_{j}(x(t)) \triangleq \frac{\theta_{j}^{U}(x(t))}{\sum_{l=1}^{p}\left(\theta_{l}^{L}(x(t))+\theta_{l}^{U}(x(t))\right)},
\end{aligned}
$$

and $\underline{K}_{j}$ and $\bar{K}_{j}$ are the controller gains associated with the lower and upper membership grades. By the above relations, $\theta_{i}(x(t)), \underline{\theta}_{i}(x(t))$, and $\bar{\theta}_{i}(x(t))$ satisfy the following conditions:

$$
\begin{aligned}
\sum_{i=1}^{p} \theta_{i}(x(t)) & =\sum_{j=1}^{p}\left(\underline{\theta}_{j}(x(t))+\bar{\theta}_{j}(x(t))\right)=1, \\
0 & \leq \alpha_{i} \leq \theta_{i}(x(t)) \leq \beta_{i} \leq 1, \\
0 & \leq \underline{\alpha}_{i} \leq \underline{\theta}_{i}(x(t)) \leq \underline{\beta}_{i} \leq 1, \\
0 & \leq \bar{\alpha}_{i} \leq \bar{\theta}_{i}(x(t)) \leq \bar{\beta}_{i} \leq 1, \\
0 & \leq \underline{\theta}_{i}(x(t))+\bar{\theta}_{i}(x(t)) \leq 1, \\
0 & \leq \theta_{i}(x(t))\left(\underline{\theta}_{j}(x(t))+\bar{\theta}_{j}(x(t))\right), \\
\underline{\theta}_{i}(x(t)) & \leq \bar{\theta}_{i}(x(t)),
\end{aligned}
$$

where $\alpha_{i}, \underline{\alpha}_{i}, \bar{\alpha}_{i}, \beta_{i}, \underline{\beta}_{i}$, and $\bar{\beta}_{i}$ are real constant values. Henceforth, for a simple description, we use the following notations: $\theta_{i}(x(t)) \triangleq \theta_{i}, \underline{\theta}_{i}(x(t)) \triangleq \underline{\theta}_{i}$, and $\bar{\theta}_{i}(x(t)) \triangleq \bar{\theta}_{i}$. The resulting closed-loop system under (4) is represented as follows:

$$
\begin{aligned}
\dot{x}(t) & =\sum_{i=1}^{p} \theta_{i}\left\{A_{i} x(t)+B_{i} \sum_{j=1}^{p}\left(\underline{\theta}_{j} \underline{K}_{j}+\bar{\theta}_{j} \bar{K}_{j}\right) x(t)\right\} \\
& =\left\{\sum_{i=1}^{p} \theta_{i} A_{i}+\sum_{i=1}^{p} \sum_{j=1}^{p}\left(\theta_{i} \underline{\theta}_{j} B_{i} \underline{K}_{j}+\theta_{i} \bar{\theta}_{j} B_{i} \bar{K}_{j}\right)\right\} x(t) .
\end{aligned}
$$




\section{Stabilization Conditions}

Theorem 1. Suppose that matrices $R_{0}, S_{0}, R_{i}, \underline{R}_{i}, \bar{R}_{i}, S_{i}, \underline{S}_{i}$, $\bar{S}_{i}, X_{i}, Y_{i}, Z_{i}, \Pi_{i}, \Xi_{i j}, \Lambda_{i} \in \mathscr{R}^{n \times n}, \underline{L}_{i}, \bar{L}_{i} \in \mathscr{R}^{m \times n}$, and a positive definite matrix $\bar{P} \in \mathscr{R}^{n \times n}$ exist such that, for $i, j=\{1,2, \ldots, p\}$,

$$
\begin{aligned}
X_{i}+X_{i}^{T} & >0, \\
Y_{i}+Y_{i}^{T} & >0, \\
Z_{i}+Z_{i}^{T} & >0,
\end{aligned}
$$

$$
\Pi_{i}+\Pi_{i}^{T}>0,
$$$$
\Xi_{i j}+\Xi_{i j}^{T}>0 \text {, }
$$$$
\Lambda_{i}+\Lambda_{i}^{T}>0 \text {, }
$$$$
\Sigma<0,
$$

$$
\begin{aligned}
& \Sigma=\left[\begin{array}{c|cccc|cccc|cccc}
\mathscr{L}_{0} & \mathscr{L}_{(0,1)} & \mathscr{L}_{(0,2)} & \cdots & \mathscr{L}_{(0, p)} & \mathscr{L}_{(0,1)} & \mathscr{L}_{(0,2)} & \cdots & \underline{L}_{(0, p)} & \overline{\mathscr{L}}_{(0,1)} & \overline{\mathscr{L}}_{(0,2)} & \cdots & \overline{\mathscr{L}}_{(0, p)} \\
\hline(*) & \Delta_{(1,1)} & \mathscr{L}_{(1,2)} & \cdots & \mathscr{L}_{(1, p)} & \underline{\Phi}_{(1,1)} & \cdots & \cdots & \underline{\Phi}_{(1, p)} & \bar{\Phi}_{(1,1)} & \cdots & \cdots & \bar{\Phi}_{(1, p)} \\
(*) & (*) & \ddots & & \vdots & \vdots & \ddots & & \vdots & \vdots & \ddots & & \vdots \\
(*) & (*) & (*) & \ddots & \vdots & \vdots & & \ddots & \vdots & \vdots & & \ddots & \vdots \\
(*) & (*) & (*) & (*) & \Delta_{(p, p)} & \underline{\Phi}_{(p, 1)} & \cdots & \cdots & \underline{\Phi}_{(p, p)} & \bar{\Phi}_{(p, 1)} & \ldots & \ldots & \Phi_{(p, p)} \\
\hline(*) & (*) & (*) & (*) & (*) & \underline{\Delta}_{(1,1)} & \mathscr{L}_{(1,2)} & \cdots & \underline{\mathscr{L}}_{(1, p)} & \widetilde{\Delta}_{(1,1)} & \cdots & \cdots & \Phi_{(1, p)} \\
(*) & (*) & (*) & (*) & (*) & (*) & \ddots & & \vdots & \vdots & \ddots & & \vdots \\
(*) & (*) & (*) & (*) & (*) & (*) & (*) & \ddots & \vdots & \vdots & & \ddots & \vdots \\
(*) & (*) & (*) & (*) & (*) & (*) & (*) & (*) & \underline{\Delta}_{(p, p)} & \widetilde{\Phi}_{(p, 1)} & \cdots & \cdots & \widetilde{\Delta}_{(p, p)} \\
\hline(*) & (*) & (*) & (*) & (*) & (*) & (*) & (*) & (*) & \bar{\Delta}_{(1,1)} & \overline{\mathscr{L}}_{(1,2)} & \cdots & \overline{\mathscr{L}}_{(1, p)} \\
(*) & (*) & (*) & (*) & (*) & (*) & (*) & (*) & (*) & (*) & \ddots & & \vdots \\
(*) & (*) & (*) & (*) & (*) & (*) & (*) & (*) & (*) & (*) & (*) & \ddots & \vdots \\
(*) & (*) & (*) & (*) & (*) & (*) & (*) & (*) & (*) & (*) & (*) & (*) & \bar{\Delta}_{(p, p)}
\end{array}\right], \\
& \mathscr{L}_{0}=\left(R_{0}+R_{0}^{T}\right)+\left(S_{0}+S_{0}^{T}\right)-\sum_{i=1}^{p} \alpha_{i} \beta_{i}\left(X_{i}+X_{i}^{T}\right)-\sum_{i=1}^{p} \underline{\alpha}_{i} \underline{\beta}_{i}\left(Y_{i}+Y_{i}^{T}\right)-\sum_{i=1}^{p} \bar{\alpha}_{i} \bar{\beta}_{i}\left(Z_{i}+Z_{i}^{T}\right), \\
& \mathscr{L}_{(0, i)}=A_{i} \bar{P}+R_{i}+S_{i}-R_{0}+\left(\alpha_{i}+\beta_{i}\right) X_{i}, \\
& \underline{\mathscr{L}}_{(0, i)}=\underline{R}_{i}+\underline{S}_{i}-S_{0}+\left(\underline{\alpha}_{i}+\underline{\beta}_{i}\right) Y_{i}+\Pi_{i} \text {, } \\
& \overline{\mathscr{L}}_{(0, i)}=\bar{R}_{i}+\bar{S}_{i}-S_{0}+\left(\bar{\alpha}_{i}+\bar{\beta}_{i}\right) Z_{i}+\Pi_{i} \text {, } \\
& \Delta_{(i, i)}=-\left(R_{i}+R_{i}^{T}\right)-\left(X_{i}+X_{i}^{T}\right) \text {, } \\
& \underline{\Delta}_{(i, i)}=-\left(\underline{S}_{i}+\underline{S}_{i}^{T}\right)-\left(Y_{i}+Y_{i}^{T}\right)-\left(\Pi_{i}+\Pi_{i}^{T}\right)-\left(\Lambda_{i}+\Lambda_{i}^{T}\right), \\
& \bar{\Delta}_{(i, i)}=-\left(\bar{S}_{i}+\bar{S}_{i}^{T}\right)-\left(Z_{i}+Z_{i}^{T}\right)-\left(\Pi_{i}+\Pi_{i}^{T}\right)+\Lambda_{i}+\Lambda_{i}^{T}, \\
& \widetilde{\Delta}_{(i, i)}=-\left(\bar{S}_{i}^{T}+\underline{S}_{i}\right)-2 \Pi_{i}, \\
& \underline{\Phi}_{(i, j)}=B_{i} \underline{L}_{j}-\left(S_{i}+\underline{R}_{j}\right)+\Xi_{i j}, \\
& \bar{\Phi}_{(i, j)}=B_{i} \bar{L}_{j}-\left(S_{i}+\bar{R}_{j}\right)+\Xi_{i j} \text {, } \\
& \mathscr{L}_{(i, j)}=-\left(R_{i}+R_{j}\right),
\end{aligned}
$$




$$
\begin{aligned}
& \underline{\mathscr{L}}_{(i, j)}=-\left(\underline{S}_{i}+\underline{S}_{j}\right), \\
& \overline{\mathscr{L}}_{(i, j)}=-\left(\bar{S}_{i}+\bar{S}_{j}\right), \\
& \widetilde{\Phi}_{(i, j)}=-\left(\underline{S}_{i}+\bar{S}_{j}\right) .
\end{aligned}
$$

Then, the closed-loop system (13) is asymptotically stable and the corresponding fuzzy controller is given by $\underline{K}_{j}=\underline{L}_{j} \bar{P}^{-1}$ and $\bar{K}_{j}=\bar{L}_{j} \bar{P}^{-1}$.

Proof. Choose a quadratic Lyapunov function, $V(t)=$ $x^{T}(t) P x(t)$, where $P$ is a positive definite matrix. Then the time derivative of $V(x(t))$ is given by

$$
\begin{aligned}
\dot{V}(t) & =2 x(t)^{T} P \dot{x}(t)=2 x(t)^{T} \\
\cdot & P\left\{\sum_{i=1}^{p} \theta_{i} A_{i}+\sum_{i=1}^{p} \sum_{j=1}^{p}\left(\theta_{i} \underline{\theta}_{j} B_{i} \underline{K}_{j}+\theta_{i} \bar{\theta}_{j} B_{i} \bar{K}_{j}\right)\right\} \\
\cdot & x(t) .
\end{aligned}
$$

For the asymptotic stability of (13), the stability criterion is given as $\dot{V}(t)<0$. It leads to the following condition:

$$
\begin{aligned}
& \operatorname{Sym}\left(\sum_{i=1}^{p} \theta_{i} P A_{i}+\sum_{i=1}^{p} \sum_{j=1}^{p}\left(\theta_{i} \underline{\theta}_{j} P B_{i} \underline{K}_{j}+\theta_{i} \bar{\theta}_{j} P B_{i} \bar{K}_{j}\right)\right) \\
& \quad<0 .
\end{aligned}
$$

Multiplying both sides of (17) by $P^{-1}$ yields

$$
\operatorname{Sym}\left(\sum_{i=1}^{p} \theta_{i} A_{i} \bar{P}+\sum_{i=1}^{p} \sum_{j=1}^{p}\left(\theta_{i} \underline{\theta}_{j} B_{i} \underline{L}_{j}+\theta_{i} \bar{\theta}_{j} B_{i} \bar{L}_{j}\right)\right)<0,
$$

where $\bar{P}=P^{-1}, \underline{L}_{j}=\underline{K}_{j} \bar{P}$, and $\bar{L}_{j}=\bar{K}_{j} \bar{P}$. We can then represent (18) as the following form:

$$
\begin{aligned}
\mathscr{M}= & \sum_{i=1}^{p} \theta_{i}\left(M_{(0, i)}+M_{(0, i)}^{T}\right) \\
& +\sum_{i=1}^{p} \sum_{j=1}^{p} \theta_{i} \underline{\theta}_{j}\left(\underline{M}_{(i, j)}+\underline{M}_{(i, j)}^{T}\right) \\
& +\sum_{i=1}^{p} \sum_{j=1}^{p} \theta_{i} \bar{\theta}_{j}\left(\bar{M}_{(i, j)}+\bar{M}_{(i, j)}^{T}\right)<0,
\end{aligned}
$$

where $M_{(0, i)}=A_{i} \bar{P}, \underline{M}_{(i, j)}=B_{i} \underline{L}_{j}$, and $\bar{M}_{(i, j)}=B_{i} \bar{L}_{j}$.
For the relaxation technique using the relationship among fuzzy weighting functions and their lower and upper membership grades, we can convert conditions (6)-(12) for all $i \in[1, p]$, respectively, into

$$
\begin{aligned}
& 0 \equiv C_{1}=\left[\begin{array}{c}
I \\
\theta_{1} I \\
\vdots \\
\theta_{p} I \\
\underline{\theta}_{1} I \\
\vdots \\
\underline{\theta}_{p} I \\
\bar{\theta}_{1} I \\
\vdots \\
-I \\
\bar{\theta}_{p} I
\end{array}\right]^{T}\left[\begin{array}{cc}
I & I \\
-I & 0 \\
0 & -I \\
\vdots & \vdots \\
0 & -I \\
0 & -I \\
\vdots & \vdots \\
0 & -I
\end{array}\right]\left[\begin{array}{cc}
R_{0}^{T} & S_{0}^{T} \\
R_{1}^{T} & S_{1}^{T} \\
\vdots & \vdots \\
R_{p}^{T} & S_{p}^{T} \\
\underline{R}_{1} & \underline{S}_{1} \\
\vdots & \vdots \\
\underline{R}_{p} & \underline{S}_{p} \\
\bar{R}_{1} & \bar{S}_{1} \\
\vdots & \vdots \\
\bar{R}_{p} & \bar{S}_{p}
\end{array}\right]^{T}\left[\begin{array}{c}
I \\
\theta_{1} I \\
\vdots \\
\theta_{p} I \\
\underline{\theta}_{p} I \\
\bar{\theta}_{1} I \\
\vdots \\
\bar{\theta}_{p} I
\end{array}\right], \\
& 0 \leq C_{2 i}=-\left(\theta_{i}-\alpha_{i}\right)\left(\theta_{i}-\beta_{i}\right)\left(X_{i}+X_{i}^{T}\right), \\
& 0 \leq C_{3 i}=-\left(\underline{\theta}_{i}-\underline{\alpha}_{i}\right)\left(\underline{\theta}_{i}-\underline{\beta}_{i}\right)\left(Y_{i}+Y_{i}^{T}\right), \\
& 0 \leq C_{4 i}=-\left(\bar{\theta}_{i}-\bar{\alpha}_{i}\right)\left(\bar{\theta}_{i}-\bar{\beta}_{i}\right)\left(Z_{i}+Z_{i}^{T}\right), \\
& 0 \leq C_{5 i}=-\left(\underline{\theta}_{i}+\bar{\theta}_{i}\right)\left(\underline{\theta}_{i}+\bar{\theta}_{i}-1\right)\left(\Pi_{i}+\Pi_{i}^{T}\right), \\
& 0 \leq C_{6 i j}=\theta_{i}\left(\underline{\theta}_{j}+\bar{\theta}_{j}\right)\left(\Xi_{i j}+\Xi_{i j}^{T}\right), \\
& 0 \leq C_{7 i}=\left(\bar{\theta}_{i}^{2}-\underline{\theta}_{i}^{2}\right)\left(\Lambda_{i}+\Lambda_{i}^{T}\right),
\end{aligned}
$$

where condition (20) is from $\sum_{i=1}^{p} \theta_{i} I-I=\sum_{i=1}^{p}\left(\underline{\theta}_{i}+\bar{\theta}_{i}\right) I-$ $I=0$ and conditions (21)-(26) are from (7)-(12), respectively. Here, the matrices $R_{0}, R_{i}, \underline{R}_{i}, \bar{R}_{i}, X_{i}, Y_{i}, Z_{i}, \Pi_{i}, \Xi_{i}$, and $\Lambda_{i}$ are slack variables to reduce conservatism of (19).

Then, merging conditions (20)-(26) gives

$$
\begin{aligned}
\mathcal{N}= & C_{1}+C_{1}^{T}+\sum_{i=1}^{p} C_{2 i}+\sum_{i=1}^{p} C_{3 i}+\sum_{i=1}^{p} C_{4 i}+\sum_{i=1}^{p} C_{5 i} \\
& +\sum_{i=1}^{p} \sum_{j=1}^{p} C_{6 i j}+\sum_{i=1}^{p} C_{7 i} \geq 0 .
\end{aligned}
$$


To derive LMI condition, (27) can be represented as the following form:

$$
\begin{aligned}
0 \leq & \mathcal{N} \\
= & N_{0}+\sum_{i=1}^{p} \theta_{i}\left(N_{(0, i)}+N_{(0, i)}^{T}\right)+\sum_{i=1}^{p} \underline{\theta}_{i}\left(\underline{N}_{(0, i)}+\underline{N}_{(0, i)}^{T}\right) \\
& +\sum_{i=1}^{p} \bar{\theta}_{i}\left(\bar{N}_{(0, i)}+\bar{N}_{(0, i)}^{T}\right)+\sum_{i=1}^{p} \theta_{i}^{2} N_{(i, i)}+\sum_{i=1}^{p} \underline{\theta}_{i}^{2} \underline{N}_{(i, i)} \\
& +\sum_{i=1}^{p} \bar{\theta}_{i}^{2} \bar{N}_{(i, i)}+\sum_{i=1}^{p} \underline{\theta}_{i} \bar{\theta}_{i}\left(\widetilde{T}_{(i, i)}+\widetilde{T}_{(i, i)}^{T}\right) \\
& +\sum_{i=1}^{p} \sum_{j=1}^{p} \theta_{i} \underline{\theta}_{j}\left(\underline{T}_{(i, j)}+\underline{T}_{(i, j)}^{T}\right) \\
& +\sum_{i=1}^{p} \sum_{j=1}^{p} \theta_{i} \bar{\theta}_{j}\left(\bar{T}_{(i, j)}+\bar{T}_{(i, j)}^{T}\right) \\
& +\sum_{i=1}^{p-1} \sum_{j=i+1}^{p} \theta_{i} \theta_{j}\left(N_{(i, j)}+N_{(i, j)}^{T}\right) \\
& +\sum_{i=1}^{p-1} \sum_{j=i+1}^{p} \theta_{i} \underline{\theta}_{j}\left(\underline{N}_{(i, j)}+\underline{N}_{(i, j)}^{T}\right) \\
& +\sum_{i=1}^{p-1} \sum_{j=i+1}^{p} \theta_{i} \bar{\theta}_{j}\left(\bar{N}_{(i, j)}+\bar{N}_{(i, j)}^{T}\right) \\
& +\sum_{i=1}^{p} \sum_{j=1, j \neq i}^{p} \underline{\theta}_{i} \bar{\theta}_{j}\left(\widetilde{T}_{(i, j)}+\widetilde{T}_{(i, j)}^{T}\right) \\
&
\end{aligned}
$$

where

$$
\begin{aligned}
N_{0}= & R_{0}+S_{0}+R_{0}^{T}+S_{0}^{T}-\sum_{i=1}^{p} \alpha_{i} \beta_{i}\left(X_{i}+X_{i}^{T}\right) \\
& -\sum_{i=1}^{p} \underline{\alpha}_{i} \underline{\beta}_{i}\left(Y_{i}+Y_{i}^{T}\right)-\sum_{i=1}^{p} \bar{\alpha}_{i} \bar{\beta}_{i}\left(Z_{i}+Z_{i}^{T}\right), \\
N_{(0, i)}= & R_{i}+S_{i}-R_{0}+\left(\alpha_{i}+\beta_{i}\right) X_{i}, \\
\underline{N}_{(0, i)}= & \underline{R}_{i}+\underline{S}_{i}-S_{0}+\left(\underline{\alpha}_{i}+\underline{\beta}_{i}\right) Y_{i}+\Pi_{i}, \\
\bar{N}_{(0, i)}= & \bar{R}_{i}+\bar{S}_{i}-S_{0}+\left(\bar{\alpha}_{i}+\bar{\beta}_{i}\right) Z_{i}+\Pi_{i}, \\
N_{(i, i)}= & -\left(R_{i}+R_{i}^{T}\right)-\left(X_{i}+X_{i}^{T}\right), \\
\underline{N}_{(i, i)}= & -\left(\underline{S}_{i}+\underline{S}_{i}^{T}\right)-\left(Y_{i}+Y_{i}^{T}\right)-\left(\Pi_{i}+\Pi_{i}^{T}\right) \\
& -\left(\Lambda_{i}+\Lambda_{i}^{T}\right), \\
\bar{N}_{(i, i)}= & -\left(\bar{S}_{i}+\bar{S}_{i}^{T}\right)-\left(Z_{i}+Z_{i}^{T}\right)-\left(\Pi_{i}+\Pi_{i}^{T}\right)+\Lambda_{i} \\
& +\Lambda_{i}^{T},
\end{aligned}
$$

$$
\begin{aligned}
& \widetilde{T}_{(i, i)}=-\left(\bar{S}_{i}^{T}+\underline{S}_{i}\right)-2 \Pi_{i}, \\
& \underline{T}_{(i, j)}=-\left(S_{i}+\underline{R}_{j}\right)+\Xi_{i j}, \\
& \bar{T}_{(i, j)}=-\left(S_{i}+\bar{R}_{j}\right)+\Xi_{i j}, \\
& N_{(i, j)}=-\left(R_{i}+R_{j}\right), \\
& \underline{N}_{(i, j)}=-\left(\underline{S}_{i}+\underline{S}_{j}\right), \\
& \bar{N}_{(i, j)}=-\left(\bar{S}_{i}+\bar{S}_{j}\right), \\
& \widetilde{T}_{(i, j)}=-\left(\underline{S}_{i}+\bar{S}_{j}\right) .
\end{aligned}
$$

The $\mathcal{S}$-procedure enables the condition in (19) subject to (28) to be expressed as

$$
\mathscr{L}=\mathscr{M}+\mathscr{N}<0,
$$

which can be converted into

$$
\mathscr{L}=\Theta^{T} \Sigma \Theta<0,
$$

where

$$
\Theta=\left[\begin{array}{lllllll}
I \mid \theta_{1} & \cdots & \theta_{p} \mid \underline{\theta}_{1} & \cdots & \underline{\theta}_{p} \mid \bar{\theta}_{1} & \cdots & \bar{\theta}_{p}
\end{array}\right]^{T} .
$$

Remark 2. The proposed LMIs are not always feasible for all type 2 T-S fuzzy system. However, the proposed method can guarantee a larger feasible solution set than the previous studies because we use the relationship among fuzzy weighting functions and their lower and upper membership grades and the proposed controller is individually dependent on the lower and upper membership grades.

\section{Numerical Examples}

Example 1. Let us consider a simple fuzzy model [21] in the following format:

Plant Rule $i: \quad$ IF $x_{1}(t)$ is $\widetilde{\mathscr{F}}_{i 1}$,

$$
\begin{array}{r}
\text { THEN } \dot{x}(t)=A_{i} x(t)+B_{i} u(t), \\
i=1,2,3,
\end{array}
$$

where

$$
\begin{aligned}
& A_{1}=\left[\begin{array}{cc}
2.78 & -5.63 \\
0.01 & 0.33
\end{array}\right], \\
& A_{2}=\left[\begin{array}{cc}
0.2 & -3.22 \\
0.35 & 0.12
\end{array}\right], \\
& A_{3}=\left[\begin{array}{cc}
-a & -6.63 \\
0.45 & 0.15
\end{array}\right],
\end{aligned}
$$




$$
\begin{aligned}
& B_{1}=\left[\begin{array}{c}
2 \\
-1
\end{array}\right], \\
& B_{2}=\left[\begin{array}{l}
8 \\
0
\end{array}\right], \\
& B_{3}=\left[\begin{array}{c}
-b+6 \\
-1
\end{array}\right],
\end{aligned}
$$

$$
\begin{aligned}
& \theta_{1}^{U}=\bar{\mu}_{\widetilde{F}_{11}}\left(x_{1}(t)\right)=0.95-\left(\frac{0.925}{\left(1+e^{-\left(\left(x_{1}+4.5\right) / 8\right)}\right)}\right), \\
& \theta_{3}^{U}=\bar{\mu}_{\widetilde{F}_{31}}\left(x_{1}(t)\right)=0.025+\left(\frac{0.925}{\left(1+e^{-\left(\left(x_{1}-4.5\right) / 8\right)}\right)}\right), \\
& \theta_{2}^{U}=\bar{\mu}_{\widetilde{F}_{21}}\left(x_{1}(t)\right)=1-\theta_{1}^{L}-\theta_{3}^{L} .
\end{aligned}
$$

where $14 \leq a \leq 30$ and $20 \leq b \leq 35$. The above model is assumed operating in the domain of $x_{1}(t) \in\left[\begin{array}{ll}-10 & 10\end{array}\right]^{T}$. The lower and upper membership functions are chosen as

$$
\begin{aligned}
& \theta_{1}^{L}=\underline{\mu}_{\widetilde{F}_{11}}\left(x_{1}(t)\right)=0.95-\left(\frac{0.925}{\left(1+e^{-\left(\left(x_{1}+3.5\right) / 8\right)}\right)}\right), \\
& \theta_{3}^{L}=\underline{\mu}_{\widetilde{F}_{31}}\left(x_{1}(t)\right)=0.025+\left(\frac{0.925}{\left(1+e^{-\left(\left(x_{1}-3.5\right) / 8\right)}\right)}\right), \\
& \theta_{2}^{L}=\underline{-}_{\widetilde{F}_{21}}\left(x_{1}(t)\right)=1-\theta_{1}^{U}-\theta_{3}^{U},
\end{aligned}
$$

$$
\dot{x}_{2}(t)=\frac{g \sin \left(x_{1}(t)\right)-a m_{p} L x_{2}^{2}(t) \sin \left(2 x_{1}(t)\right) / 2-a \cos \left(x_{1}(t)\right) u(t)}{4 L / 3-a m_{p} L \cos ^{2}\left(x_{1}(t)\right)}
$$

where $x_{1}(t)$ is the angular displacement of the pendulum; $g=$ $9.8 \mathrm{~m} / \mathrm{s}^{2} ; m_{p}$ and $M_{c}$ are uncertain in $m_{p_{\text {min }}}=2 \leq m_{p} \leq 5=$ $m_{p_{\max }}$ and $M_{c \text { min }}=8 \leq M_{c} \leq 18=M_{c \max }$, respectively; $a=1 /\left(m_{p}+M_{c}\right) ; 2 L=1 \mathrm{~m}$; and $u(t)$ is the force applied to the cart. From [21], we can obtain a plant rule to describe the inverted pendulum subject to parameter uncertainties in the following format:

Plant Rule $i: \quad$ IF $x_{1}(t)$ is $\widetilde{\mathscr{F}}_{i 1}$,

$$
\begin{aligned}
& \text { IF } x_{1}(t) \text { is } \widetilde{\mathscr{F}}_{i 2} \\
& \text { THEN } \dot{x}(t)=A_{i} x(t)+B_{i} u(t), \\
& \qquad i=1,2,3,4,
\end{aligned}
$$

where

$$
\begin{aligned}
& A_{1}=A_{2}=\left[\begin{array}{cc}
0 & 1 \\
f_{1 \text { min }} & 0
\end{array}\right], \\
& A_{3}=A_{4}=\left[\begin{array}{cc}
0 & 1 \\
f_{1 \max } & 0
\end{array}\right], \\
& B_{1}=B_{3}=\left[\begin{array}{c}
0 \\
f_{2 \min }
\end{array}\right], \\
& B_{2}=B_{4}=\left[\begin{array}{c}
0 \\
f_{2 \max }
\end{array}\right],
\end{aligned}
$$

For Theorem $1, \alpha_{i}=0, \beta_{i}=1, \underline{\alpha}_{1}=\underline{\alpha}_{3}=0.154, \underline{\alpha}_{2}=0.164$, $\underline{\beta}_{1}=\underline{\beta}_{3}=0.65, \underline{\beta}_{2}=0.25, \bar{\alpha}_{1}=\bar{\alpha}_{3}=0.169, \bar{\alpha}_{2}=0.204$, $\bar{\beta}_{1}=\bar{\beta}_{3}=0.67, \bar{\beta}_{2}=0.3$. Figure 1 shows the stability regions for the proposed conditions and previous conditions in Example 1 of [21]. From Figure 1, we can clearly see that the proposed conditions outperform previous one [21] in terms of a larger stability region. It is an important observation that the proposed conditions with a multigain controller and a well-organized relaxation technique achieve much better performance than those of the previous study [21].

Example 2. Let us consider an inverted pendulum model subject to parameter uncertainties, which is adapted from [26]:

$$
\begin{aligned}
& x(t)=\left[\begin{array}{ll}
x_{1}(t) & x_{2}(t)
\end{array}\right]^{T}, \\
& x_{1}(t) \in\left[\begin{array}{ll}
x_{1 \min } & x_{1 \max }
\end{array}\right]=\left[\begin{array}{ll}
-\frac{5 \pi}{12} & \frac{5 \pi}{12}
\end{array}\right], \\
& x_{2}(t) \in\left[\begin{array}{ll}
x_{2 \min } & x_{2 \max }
\end{array}\right]=\left[\begin{array}{ll}
-5 & 5
\end{array}\right], \\
& f_{1 \text { min }}=8.81 \leq f_{1}(x(t)) \leq f_{1 \max }=20.7, \\
& f_{2 \min }=-0.18 \leq f_{2}\left(x_{1}(t)\right) \leq f_{2 \max }=-0.017, \\
& f_{1}(x(t)) \\
& \quad=\frac{g-a m_{p} L x_{2}^{2}(t) \cos \left(x_{1}(t)\right)}{4 L / 3-a m_{p} L \cos ^{2}\left(x_{1}(t)\right)}\left(\frac{\sin \left(x_{1}(t)\right)}{x_{1}(t)}\right), \\
& f_{2}\left(x_{1}(t)\right)=\frac{-a \cos \left(x_{1}(t)\right)}{4 L / 3-a m_{p} L \cos ^{2}\left(x_{1}(t)\right)} .
\end{aligned}
$$

The type 2 T-S fuzzy model is represented as follows:

$$
\begin{aligned}
& \dot{x}(t)=\sum_{i=1}^{4} \theta_{i}\left(A_{i} x(t)+B_{i} u(t)\right) \\
&= \sum_{i=1}^{4}\left\{\eta(x(t)) \theta_{i}^{L}+(1-\eta(x(t))) \theta_{i}^{U}\right\} \\
& \cdot\left(A_{i} x(t)+B_{i} u(t)\right),
\end{aligned}
$$




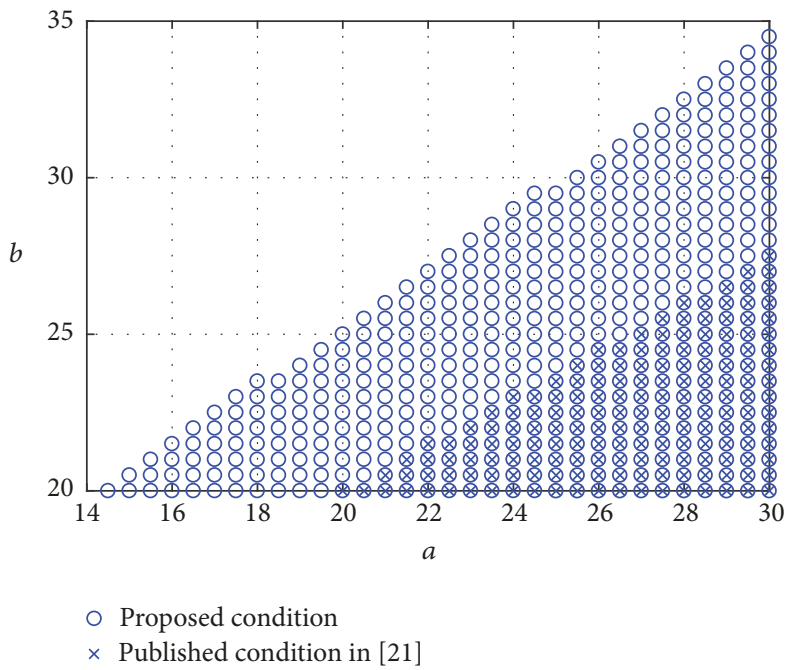

Figure 1: Stability region for the proposed ( $\circ$ ) and published conditions $(x)$.

where, for all $i$,

$$
\begin{aligned}
& \theta_{i}^{L}=\underline{\mu}_{\widetilde{F}_{i 1}}(x(t)) \times \underline{\mu}_{\widetilde{F}_{i 2}}(x(t)), \\
& \theta_{i}^{U}=\bar{\mu}_{\widetilde{F}_{i 1}}(x(t)) \times \bar{\mu}_{\widetilde{F}_{i 2}}(x(t)),
\end{aligned}
$$

and the lower and upper grades of membership for each rule are defined as follows:

$$
\begin{aligned}
& \text { (i) } x_{2}(t)=x_{2 \max }, m_{p}=m_{p_{\max }} \text { and } M_{c}=M_{c \min }: \\
& \underline{\mu}_{\widetilde{F}_{11}}(x(t))=\underline{\mu}_{\widetilde{F}_{21}}(x(t))=\frac{f_{1 \max }-f_{1}(x(t))}{f_{1 \max }-f_{1 \min }}, \\
& \underline{\mu}_{\widetilde{F}_{31}}(x(t))=\underline{\mu}_{\widetilde{F}_{41}}(x(t))=\frac{f_{1}(x(t))-f_{1 \min }}{f_{1 \max }-f_{1 \min }}
\end{aligned}
$$

(ii) $x_{2}(t)=0, m_{p}=m_{p_{\max }}$, and $M_{c}=M_{c \text { min }}$ :

$$
\begin{aligned}
& \bar{\mu}_{\widetilde{F}_{11}}(x(t))=\bar{\mu}_{\widetilde{F}_{21}}(x(t))=\frac{f_{1 \text { max }}-f_{1}(x(t))}{f_{1 \max }-f_{1 \min }}, \\
& \bar{\mu}_{\widetilde{F}_{31}}(x(t))=\bar{\mu}_{\widetilde{F}_{41}}(x(t))=\frac{f_{1}(x(t))-f_{1 \text { min }}}{f_{1 \max }-f_{1 \min }} .
\end{aligned}
$$

(iii) $m_{p}=m_{p_{\min }}$ and $M_{c}=M_{c \text { min }}$ :

$$
\begin{aligned}
& \underline{\mu}_{\widetilde{F}_{12}}\left(x_{1}(t)\right)=\underline{\mu}_{\widetilde{F}_{32}}\left(x_{1}(t)\right)=\frac{f_{2 \max }-f_{2}\left(x_{1}(t)\right)}{f_{2 \max }-f_{2 \min }}, \\
& \underline{\mu}_{\widetilde{F}_{22}}\left(x_{1}(t)\right)=\underline{\mu}_{\widetilde{F}_{42}}\left(x_{1}(t)\right)=\frac{f_{2}\left(x_{1}(t)\right)-f_{2 \min }}{f_{2 \max }-f_{2 \min }} .
\end{aligned}
$$

(iv) $m_{p}=m_{p_{\max }}$ and $M_{c}=M_{c \max }$ :

$$
\begin{aligned}
& \bar{\mu}_{\widetilde{F}_{12}}\left(x_{1}(t)\right)=\bar{\mu}_{\widetilde{F}_{32}}\left(x_{1}(t)\right)=\frac{f_{2 \max }-f_{2}\left(x_{1}(t)\right)}{f_{2 \max }-f_{2 \min }}, \\
& \bar{\mu}_{\widetilde{F}_{22}}\left(x_{1}(t)\right)=\bar{\mu}_{\widetilde{F}_{42}}\left(x_{1}(t)\right)=\frac{f_{2}\left(x_{1}(t)\right)-f_{2 \min }}{f_{2 \max }-f_{2 \min }} .
\end{aligned}
$$
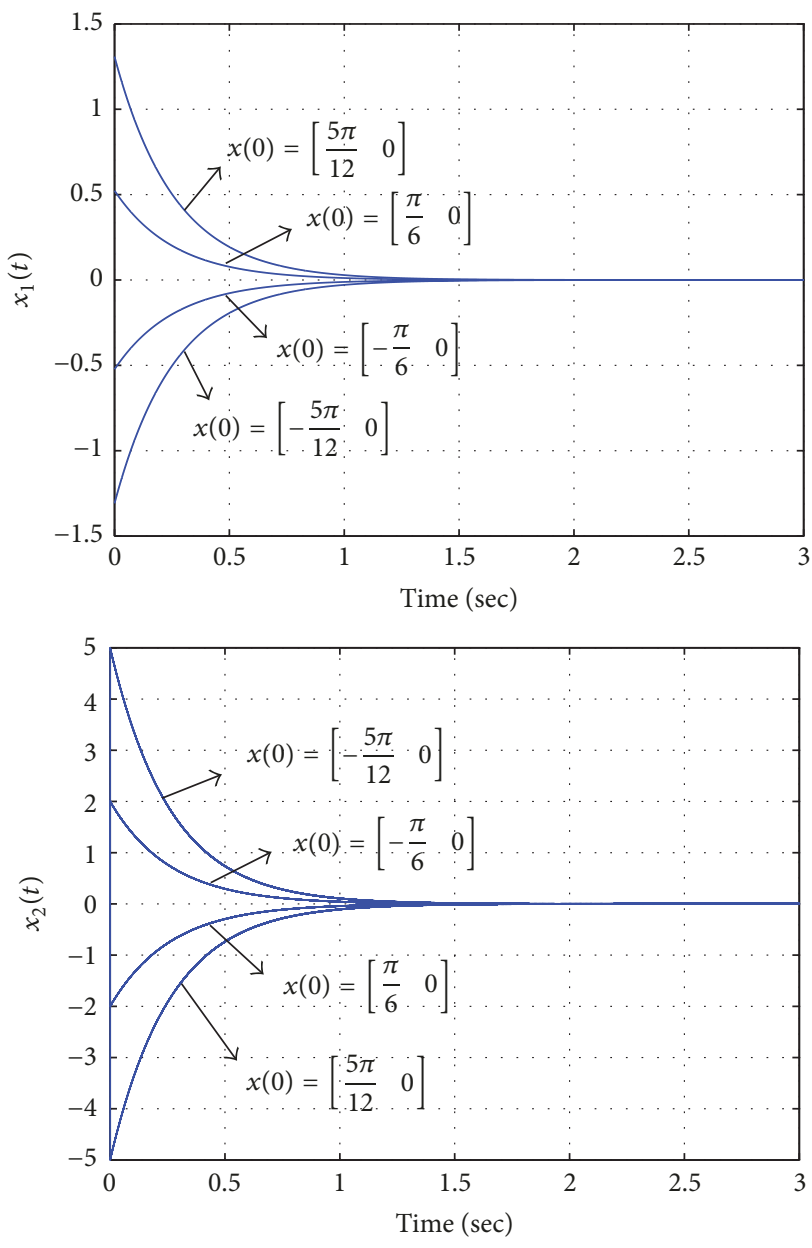

FIGURE 2: The trajectories of state $\left(m_{p}=m_{p_{\text {min }}}, M_{c}=M_{c \text { min }}\right)$.

For Theorem 1, $\alpha_{i}=0, \beta_{i}=1, \underline{\alpha}_{i}=0, \underline{\beta}_{i}=0.5, \bar{\alpha}_{1}=0$, $\bar{\beta}_{i}=0.5$. Let us demonstrate the validity of the proposed conditions for the type 2 T-S fuzzy model. Figure 2 shows the trajectories of the states with $m_{p}=m_{p_{\text {min }}}$ and $M_{c}=$ $M_{c \text { min }}$ under various initial conditions. Figure 3 shows the trajectories of the states with $m_{p}=m_{p_{\max }}$ and $M_{c}=M_{c \max }$. From Figures 2 and 3, we can clearly see that the proposed controller can stabilize the inverted pendulum with different parameter values and is robust to parameter variations in the plant model. In addition, the proposed conditions lead to less conservative results because we use the larger mass ranges than those of [21].

\section{Conclusion}

In this paper, we proposed robust stability conditions to stabilize type 2 T-S fuzzy systems. The conditions effectively handled parameter uncertainties using lower and upper membership functions. Furthermore, by applying a multigain controller and a well-organized relaxation technique, we derived a less conservative stabilization condition in terms of LMIs than those in the literature. Our simulation results showed the effectiveness and robustness of the derived stabilization conditions. 

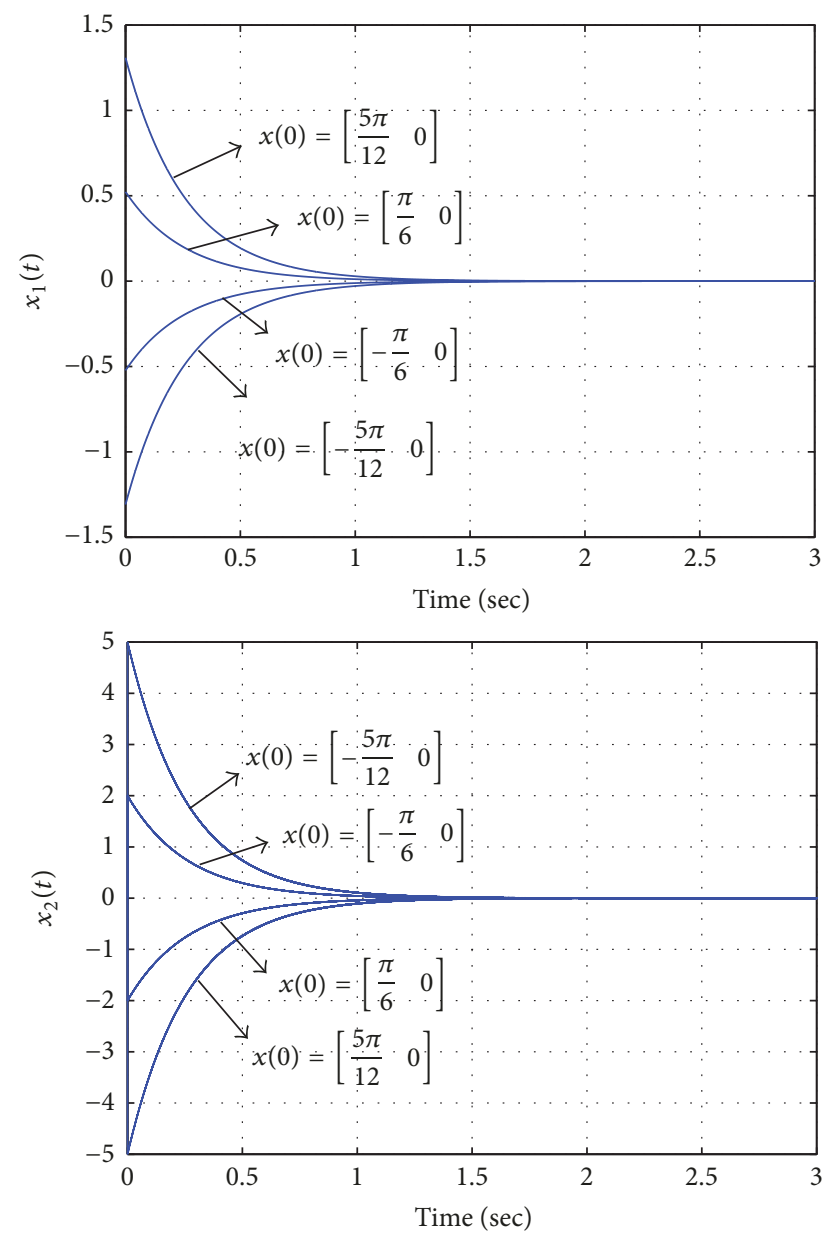

FIGURE 3: The trajectories of state $\left(m_{p}=m_{p_{\max }}, M_{c}=M_{c \max }\right)$.

\section{Conflicts of Interest}

The authors declare that there are no conflicts of interest regarding the publication of this paper.

\section{Acknowledgments}

This research was supported by Basic Science Research Program through the National Research Foundation of Korea (NRF) funded by the Ministry of Education, Science and Technology (NRF-2017R1C1B5076575).

\section{References}

[1] T. Takagi and M. Sugeno, "Fuzzy identification of systems and its applications to modeling and control," IEEE Transactions on Systems, Man, and Cybernetics, vol. 15, no. 1, pp. 116-132, 1985.

[2] L. Li, H. Peng, and Y. Yang, "Chaotic ant swarm designed T-S fuzzy system for adaptive control of dynamical systems," NeuroQuantology, vol. 6, no. 4, pp. 379-386, 2008.

[3] L. Li, Y. Yang, and H. Peng, "Fuzzy system identification via chaotic ant swarm," Chaos, Solitons \& Fractals, vol. 41, no. 1, pp. 401-409, 2009.

[4] K.-Y. Lian, J.-J. Liou, and C.-Y. Huang, "LMI-based integral fuzzy control of DC-DC converters," IEEE Transactions on Fuzzy Systems, vol. 14, no. 1, pp. 71-80, 2006.
[5] J.-W. Jung, T. H. Kim, and H. H. Choi, "Speed control of a permanent magnet synchronous motor with a torque observer: a fuzzy approach," IET Control Theory \& Applications, vol. 4, no. 12, pp. 2971-2981, 2010.

[6] C.-S. Chiu, "T-S fuzzy maximum power point tracking control of solar power generation systems," IEEE Transactions on Energy Conversion, vol. 25, no. 4, pp. 1123-1132, 2010.

[7] H. O. Wang, K. Tanaka, and M. F. Griffin, "An approach to fuzzy control of nonlinear systems: stability and design issues," IEEE Transactions on Fuzzy Systems, vol. 4, no. 1, pp. 14-23, 1996.

[8] K. Tanaka, T. Ikeda, and H. O. Wang, "Fuzzy regulators and fuzzy observers: relaxed stability conditions and LMI-based designs," IEEE Transactions on Fuzzy Systems, vol. 6, no. 2, pp. 250-265, 1998.

[9] L.-X. Li, H.-P. Peng, H.-B. Lu, and X.-P. Guan, "On feedback control of delayed chaotic system," Chinese Physics, vol. 10, no. 9, pp. 796-804, 2001.

[10] L. Li, H. Peng, H. Lu, and X. Guan, "Control and synchronization of Hénon chaotic system," Acta Physica Sinica, vol. 50, no. 4, pp. 629-632, 2001.

[11] E. Kim and H. Lee, "New approaches to relaxed quadratic stability condition of fuzzy control systems," IEEE Transactions on Fuzzy Systems, vol. 8, no. 5, pp. 523-534, 2000.

[12] C.-H. Fang, Y.-S. Liu, S.-W. Kau, L. Hong, and C.-H. Lee, "A new LMI-based approach to relaxed quadratic stabilization of T-S fuzzy control systems," IEEE Transactions on Fuzzy Systems, vol. 14, no. 3, pp. 386-397, 2006.

[13] M. C. M. Teixeira, E. Assunção, and R. G. Avellar, "On Relaxed LMI-Based Designs for Fuzzy Regulators and Fuzzy Observers," IEEE Transactions on Fuzzy Systems, vol. 11, no. 5, pp. 613-623, 2003.

[14] S. H. Kim and P. Park, " $\mathscr{H} \infty$ state-feedback-control design for discrete-time fuzzy systems using relaxation technique for parameterized LMI," IEEE Transactions on Fuzzy Systems, vol. 18, no. 5, pp. 985-993, 2010.

[15] D. Wu, "On the fundamental differences between interval type2 and type-1 fuzzy logic controllers," IEEE Transactions on Fuzzy Systems, vol. 20, no. 5, pp. 832-848, 2012.

[16] Type-2 Fuzzy Logic: Theory and Applications, vol. 223, Springer Berlin Heidelberg, Berlin, Heidelberg, 2008.

[17] H. Hagras, "Type-2 FLCs: A new generation of fuzzy controllers," IEEE Computational Intelligence Magazine, vol. 2, no. 1, pp. 30-43, 2007.

[18] J. M. Mendel, R. I. John, and F. Liu, "Interval type-2 fuzzy logic systems made simple," IEEE Transactions on Fuzzy Systems, vol. 14, no. 6, pp. 808-821, 2006.

[19] S. Coupland and R. John, "Geometric type-1 and type-2 fuzzy logic systems," IEEE Transactions on Fuzzy Systems, vol. 15, no. 1, pp. 3-15, 2007.

[20] H. A. Hagras, "A hierarchical type-2 fuzzy logic control architecture for autonomous mobile robots," IEEE Transactions on Fuzzy Systems, vol. 12, no. 4, pp. 524-539, 2004.

[21] H. K. Lam and L. D. Seneviratne, "Stability analysis of interval type-2 fuzzy-model-based control systems," IEEE Transactions on Systems, Man, and Cybernetics, Part B: Cybernetics, vol. 38, no. 3, pp. 617-628, 2008.

[22] H. K. Lam, H. Li, C. Deters, E. L. Secco, H. A. Wurdemann, and K. Althoefer, "Control design for interval type-2 fuzzy systems under imperfect premise matching," IEEE Transactions on Industrial Electronics, vol. 61, no. 2, pp. 956-968, 2014. 
[23] C.-H. Lee and F.-Y. Chang, "Interval type-2 recurrent fuzzy neural system for nonlinear systems control using stable simultaneous perturbation stochastic approximation algorithm," Mathematical Problems in Engineering, vol. 2011, Article ID 102436, 2011.

[24] R. H. Abiyev, "Credit rating using type-2 fuzzy neural networks," Mathematical Problems in Engineering, vol. 2014, Article ID 460916, 2014.

[25] M. F. Hamza, H. J. Yap, and I. A. Choudhury, "Genetic Algorithm and Particle Swarm Optimization Based Cascade Interval Type 2 Fuzzy PD Controller for Rotary Inverted Pendulum System," Mathematical Problems in Engineering, vol. 2015, Article ID 695965, 2015.

[26] X.-J. Ma and Z.-Q. Sun, "Analysis and design of fuzzy reduceddimensional observer and fuzzy functional observer," Fuzzy Sets and Systems, vol. 120, no. 1, pp. 35-63, 2001. 


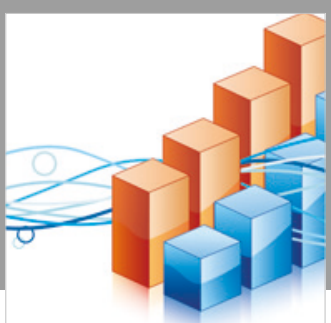

Advances in

Operations Research

\section{-n-m}
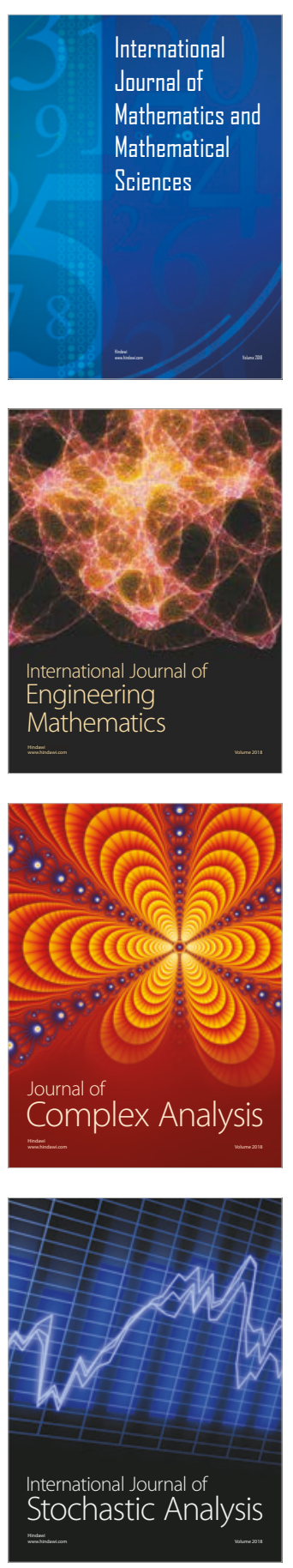
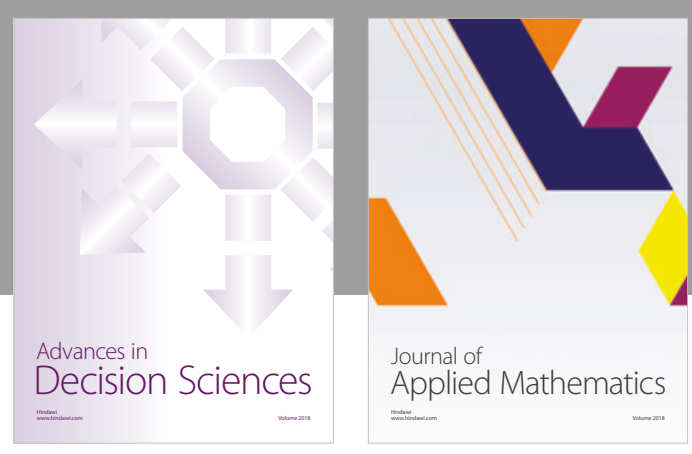

Journal of

Applied Mathematics
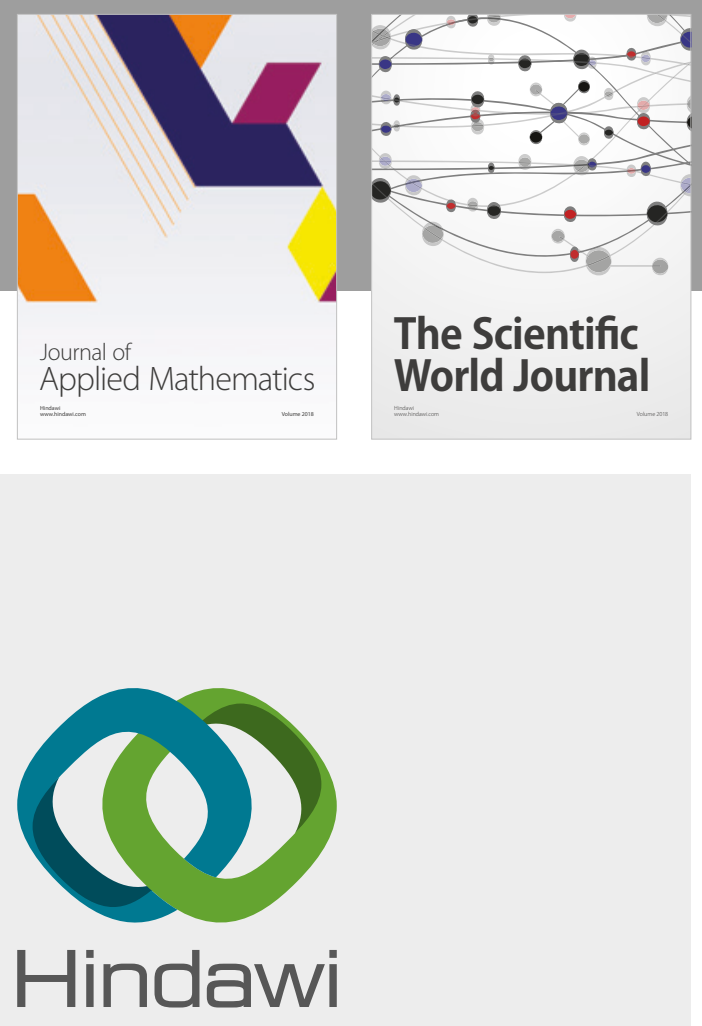

Submit your manuscripts at

www.hindawi.com

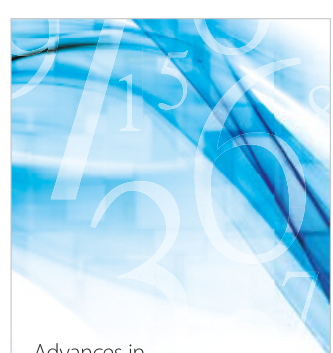

Advances in
Numerical Analysis
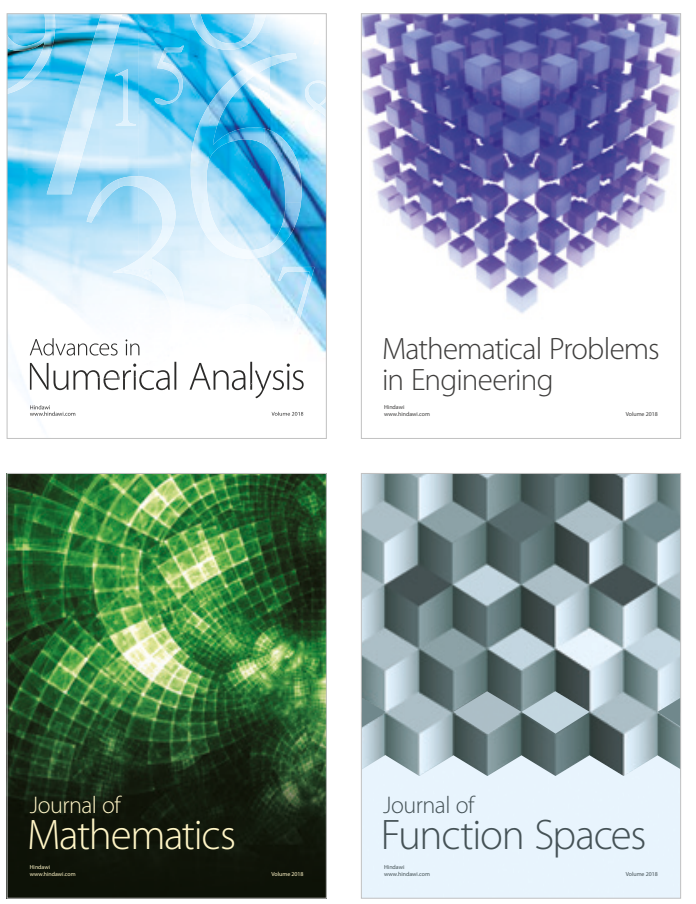

Mathematical Problems in Engineering

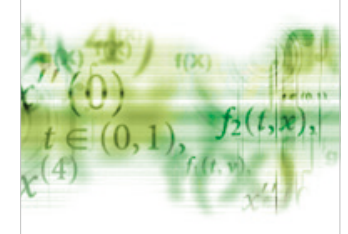

International Journal of

Differential Equations

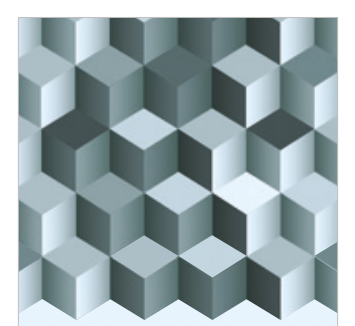

Journal of

Function Spaces

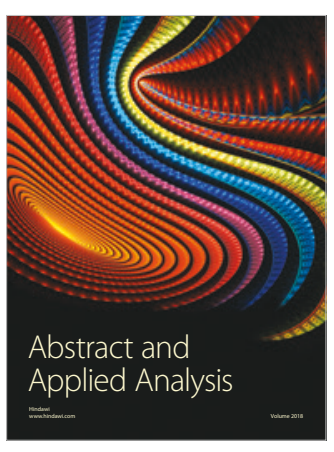

The Scientific

World Journal

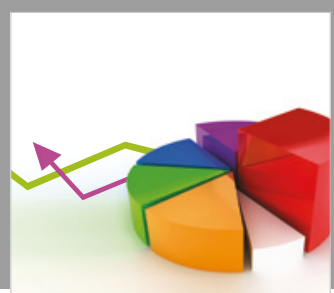

Journal of

Probability and Statistics
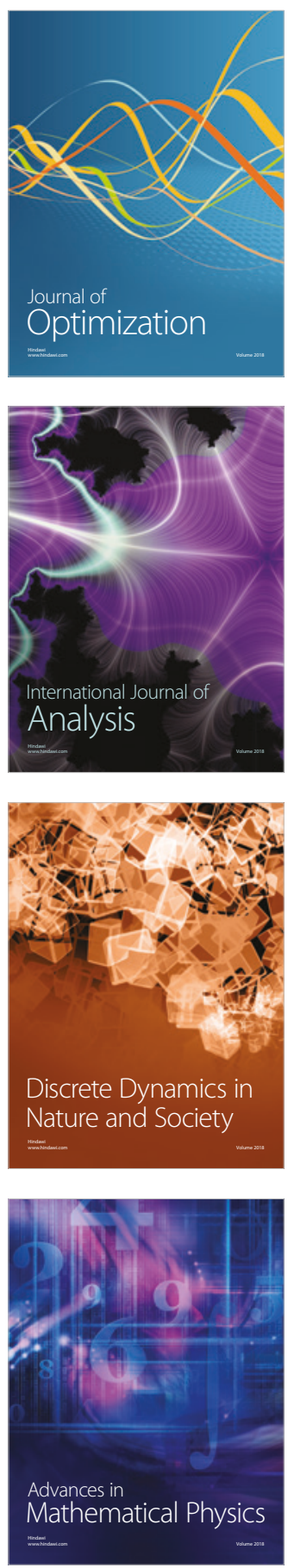